\title{
La science moderne n'est pas une Éthique
}

\author{
Alain E. Bussard
}

l existe une curieuse tendance récente, particulièrement marquée depuis la médiatisation de la science, à rendre celle-ci responsable des choix éthiques.

Il convient, d'abord, me semble-t-il, d'examiner le sens du mot "éthique " qui est le type du mot piège. Si l'on se contente de l'interprétation étymologique, la situation est claire: par sa racine grecque ethos, le mot désigne la science des mours, c'est-à-dire la morale. Cette définition de l'éthique reflète une interprétation sémantique récente. Il semble que, d'Aristote à Spinoza, l'idée d'éthique englobe un ensemble infiniment plus vaste qu'aujourd'hui. Cet ensemble contient la politique, les mœurs, l'univers et son explication, la métaphysique, etc. Dans cette acception classique du mot, notre distinction entre le bon, le vrai et le beau n'aurait aucun sens car ils sont consubstantiels. Notre interprétation du mot est donc celle d'un homme du $\mathrm{XX}^{\mathrm{e}}$ siècle, et particulièrement d'un scientifique, suivant une méthode réductrice. Pour Spinoza, notre distinction entre Science et Éthique serait évidemment absurde.

Pour un scientifique d'aujourd'hui, cela est tout à fait satisfaisant, car il se trouve, avec la morale, devant un domaine qui ne concerne pas sa propre discipline (ce qui ne veut pas dire qu'en tant qu'homme ou que citoyen elle lui soit étrangère). Si nous nous cantonnons à la définition précédente, et si nous n'introduisons pas une dimension métaphysique dans l'éthique (c'est-à-dire qui dépasse l'homme biologique), nous restons dans une notion sociologique de l'éthique qui définit celle-ci régissent une société donnée à un moment donné: règles liées à sa culture, à son histoire, à sa composition ethnique, etc. L'éthique fixe alors ce qui est bon ou mauvais, ce qui est juste et injuste, etc. Si la science est du domaine de la connaissance, l'éthique est du domaine du choix social. La science n'a pas d'aspect normatif. L'éthique, en revanche, est fondamentalement normative. Ces deux domaines ne peuvent donc, à mes yeux, absolument pas coïncider. On connaît l'exemple fameux des néopositivistes de l'Ecole de Vienne (Karnap) concernant les domaines logiques non compatibles; la question: "Napoléon est-il un nombre premier?" ne comporte pas de réponse parce qu'elle n'a pas de sens! On pourrait poser, de la même façon, la question: " le deuxième principe de la thermodynamique est-il bon ou est-il mauvais ? " Cela n'a pas de sens!

Notons, d'ailleurs, que des considérations éthiques se glissent bien rarement dans des sciences physiques, chimiques, cosmologiques, etc., mais presque uniquement en biologie et, de fait, essentiellement en biologie humaine. On observe une confusion continuelle entre la science fondamentale, la technologie et les utilisations de la technologie. La technologie, consistant à appliquer les lois découvertes par la science, n'a pas à juger de l'aspect éthique de ses applications; l'éthique n'intervient que lorsqu'il convient de décider s'il faut utiliser ces applications. Prenons un exemple à la mode, dans le domaine du test de séropositivité du SIDA. Les découvertes fondamentales de l'immunologie ont montré qu'au cours d'infections vira- les, il se développe, dans le sérum du malade, des anticorps spécifiques qui sont des marqueurs du virus (des signes de l'infection). L'immunologie appliquée a permis de mettre au point un test sérologique (Élisa) rapide, spécifique et fiable (autant qu'il est possible). Ces deux étapes ne sont pas du domaine de la morale. Il s'agit ensuite de juger s'il convient d'appliquer ce test à tous ou seulement aux groupes à risque, ou aux femmes enceintes, etc., si ces tests doivent être obligatoires ou facultatifs, etc. Ce domaine n'est plus celui de la science, mais celui de l'éthique.

Le scientifique, et même le médecin, ne sont ni plus ni moins habilités à juger de l'aspect éthique de ces décisions que n’importe quel autre citoyen. Il n'y a pas de robe prétexte qui enveloppe le savant et lui donne une priorité pour une décision qui touche la société. Son seul rôle spécifique est d'éclairer la société sur ce que les applications rendent réalisable. Le technicien indique ce qui est possible, non ce qui est bon !

Par ailleurs, il se produit, en somme, une rétroaction entre les conséquences humaines des découvertes scientifiques et les aspects éthiques (?) de celles-ci. Plus le lien est évident, plus grand est le risque d'intervention de la morale dans un ordre (celui de la connaissance) qui n'est pas le sien. Cet effet s'accroît avec l'hypermédiatisation qui affecte aujourd'hui les découvertes médicales. Les responsables de la médiatisation (journalistes et autres) étant, en général, et en France en particulier, assez ignorants et peu informés, aggravent les effets de la diffusion médiatique par des erreurs et 
des interprétations tronquées. Cette rétroaction a moins de chances de se produire (ou elle s'effectue plus tardivement) en physique, bien qu'il y ait une accélération des processus à l'époque moderne.

Par exemple, il s'est écoulé beaucoup de temps avant que les sociologues (et encore beaucoup plus de temps pour les politiques) ne s'avisent de la portée pratique du $2^{c}$ principe de la thermodynamique, qui régissait pourtant le rendement des machines thermiques et, par conséquent, allait transformer la vie de l'humanité avec le développement de la machine à vapeur. Il allait déjà falloir moins de temps pour qu'on s'avise que la fission nucléaire pouvait conduire à la réalisation de la bombe atomique et que cela provoque des crises de conscience d'Oppenheimer, par exemple. En ce qui concerne la biologie, le temps de latence entre la découverte fondamentale et surtout son application, et les conséquences sociales qui en découlent, s'est beaucoup raccourci: par exemple, les possibilités offertes par la fécondation in vitro et ses effets possibles sur le choix du sexe de l'enfant, les traitements moléculaires des maladies génétiques et l'eugénisme, ont eu un temps de latence de l'ordre de cinq à dix ans. Les événements vont encore se précipiter maintenant.

La rapidité du processus donne effectivement le vertige au lecteur attentif de la presse scientifique périodique. Comme le vertige n'est pas la condition mentale la plus appropriée pour peser les choix éthiques et que, de plus, le "citoyen éclairé" (et le scientifique d'une discipline différente de celle qui est en cause) n'ont pas les informations pertinentes, on peut s'estimer légitimement inquiet de l'avenir que la science réserve à l'humanité !

\footnotetext{
Alain E. Bussard

Professeur honoraire à l'Institut Pasteur, Vice président de l'Association "Chercheurs toujours ", 1578, route des Serres, 06570 SaintPaul, France.
} 